\title{
Leaves Shape Classification Using Curvature and Fractal Dimension
}

\author{
João B. Florindo ${ }^{1}$, André R. Backes ${ }^{2}$, and Odemir M. Bruno ${ }^{1}$ \\ 1 Instituto de Física de São Carlos (IFSC) \\ Universidade de São Paulo (USP) \\ Avenida do Trabalhador São-carlense, 400 \\ 13560-970 São Carlos SP Brazil \\ 2 Instituto de Ciências Matemáticas e de Computação (ICMC) \\ Universidade de São Paulo (USP) \\ Avenida do Trabalhador São-carlense, 400 \\ 13560-970 São Carlos SP Brazil \\ jbflorindo@ursa.ifsc.usp.br, backes@icmc.usp.br, \\ bruno@ifsc.usp.br
}

\begin{abstract}
The great biodiversity of species makes the plants classification a very complex and time-consuming task. The leaf is an important characteristic of the plant and it is present independently of season or plant maturity. The most relevant information about the leaf relies on shape. Its study enables us to discriminate a large set of species and to speed up the measures extraction process, which is traditionally performed manually. This paper presents a novel approach to leaf shape identification based on curvature complexity analysis. By using the Curvature Scale Space (CSS), a curve describing the complexity of the shape is achieved. Descriptors computed from this curve are used to classify a set of leaves shapes. Results demonstrate the potential of the technique, which overcome traditional shape analysis methods found in literature.
\end{abstract}

Keywords: shape analysis, curvature, complexity, fractal dimension.

\section{Introduction}

Shape analysis has presented a growing importance in the solution of problems related to pattern recognition and image analysis. In fact, the literature shows various works [1 [2, 3] discussing the use of shapes, mainly in classification and identification problems. Generally, these works divide shape analysis methods into 2 categories. The first category is the region-based approach, based on moment descriptors 4]. The second and, in most of the cases, a more efficient category is that of the contour-based methods. In this class of methods we find the Fourier descriptors [5], the Multi-scale Fractal Dimension [6], the Curvature Scale-Space [7, among others.

The present work studies the application of a contour-based shape analysis method to the classification of Brazilian plant species by using the contour of 
the leaf. For this, we considered the calculus of fractal dimension of the contour by the method described in [8]. Then, descriptors are computed by exploiting the richness of details of the resulting curve.

The proposed technique is validated by means of a comparison with classical literature methods such as Fourier descriptors, Zernike moments and Multi-scale Fractal Dimension. The methods are used in a plant leaf classification task. Results demonstrated an advantage of the proposed technique in terms of the precision in the classification process.

This work is divided into 6 sections, including this introduction. The following section describes the calculus of fractal dimension based on Curvature ScaleSpace technique. In the third section, we present a novel approach for obtaining descriptors related to the complexity of the shape. The fourth section describes the experiments used to validate the proposed technique, the fifth shows the results and the last section shows the final conclusions of the work.

\section{Fractal Dimension Based on Curvature}

The shape analysis literature [7] 9] shows the curvature as an important measure of a closed contour, expressing information of direction of lines tangent to the curve.

For a simplification of the mathematics, we describe the curve as a parametric vector $\boldsymbol{C}(\boldsymbol{t})=(x(u), y(u))$ and so the curvature is given by:

$$
k(t)=\frac{x^{(1)}(t) y^{(2)}(t)-x^{(2)}(t) y^{(1)}(t)}{\left(x^{(1)}(t)^{2}+y^{(1)}(t)^{2}\right)^{3 / 2}},
$$

where ${ }^{(1)}$ and ${ }^{(2)}$ represents the first and second derivatives, respectively.

In order to evaluate the curvature at different observation scales and, thus, extract a richer information about the analyzed curve, the literature shows the development of the Curvature Scale-Space technique [71011]. In this approach, a Gaussian $g(t, \sigma)$, with standard deviation $\sigma$ is applied to the contour $C(t)$ (Figure 11). As demonstrated in [8], in this case, the curvature for each scale parameter $\sigma$ can be represented by:

$$
k(t, \sigma)=\frac{X^{(1)}(t, \sigma) Y^{(2)}(t, \sigma)-X^{(2)}(t, \sigma) Y^{(1)}(t, \sigma)}{\left(X^{(1)}(t, \sigma)^{2}+Y^{(1)}(t, \sigma)^{2}\right)^{3 / 2}},
$$

where

$$
\begin{aligned}
& X(t, \sigma)=x(t) * g(t, \sigma), \\
& Y(t, \sigma)=y(t) * g(t, \sigma),
\end{aligned}
$$

The Curvature Scale Space (CSS) is composed by the set of curves obtained through the application of each value of $\sigma$.

Particularly, 8] uses the CSS concept to define the amount of curvature $S(\sigma)$ by:

$$
S(\sigma)=\sum_{t}|k(t, \sigma)|
$$


From this formula we can thus define the accumulated amount of curvature by:

$$
A(\sigma)=\sum_{i=0}^{\sigma} S(i) .
$$

In 8 , it is demonstrated that $A(\sigma)$, when varying $\sigma$, can be used as a complexity measure of the original contour. This fact, associated to the observation of a power law between $A(\sigma)$ and $\sigma$, yields to the estimation of the fractal dimension of the contour by:

$$
D=\lim _{\sigma \rightarrow 0} \frac{\log A(\sigma)}{\log \sigma} .
$$

In practice, $D$ is estimated by the slope of a straight line fitted to the curve $A(\sigma) \times \sigma$ plotted in log-log scale.

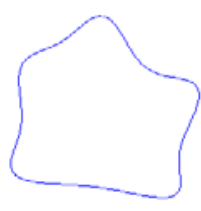

(a)

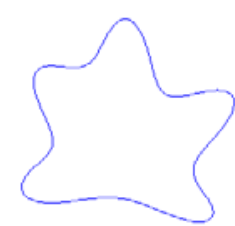

(b)

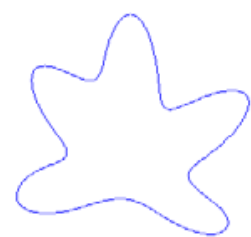

(c)

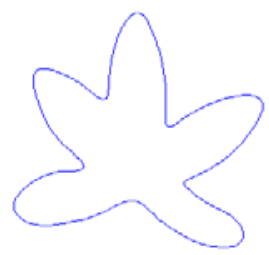

(d)

Fig. 1. Smoothing of a shape contour as the $\sigma$ parameter changes

\section{Fractal Signature}

Self-similarity in fractal objects is present at infinite scales. Otherwise, images and other real objects are limited in terms of size and resolution and, therefore, their self-similarity is limited to some scales. This limitation reflects on changes of the complexity of the image according to the scale used in the visualization, which is perceived as we study the behavior of the log-log curve computed by any fractal method. This curve cannot be completely described by using a simple line regression as used in the most fractal dimension estimation processes as it presents a richness of details along the scales which do not fit perfectly on a straight line (Figure 2);

In order to explorer this richness of details of the log-log curve, a feature vector focused on the differences of the estimated line and the real curve is proposed. Initially, a straight line which approximates the log-log curve is estimated as $y=a x+b$, where $x$ and $y$ are, respectively, the $\log$ of $\sigma$ and $A(\sigma), a$ is the slope and $b$ gives the $\mathrm{y}$-intercept of the line. This line is just an approximation of the real behavior of the log-log curve and there is an error between them. This error is related to small particularities of the shape. The difference between the estimated line and the log-log curve at a given point is defined as the simple difference $e_{i}$ :

$$
e_{i}=a \log \sigma_{i}+b-\log A\left(\sigma_{i}\right),
$$


where $\sigma_{i}$ is a value which exists in the log-log curve. Shape characterization is performed by selecting a total of $n$-first points from the log-log curve to compose a feature vector $\psi(n)$ :

$$
\psi(n)=\left[e_{1}, e_{2}, \ldots, e_{n}\right] .
$$

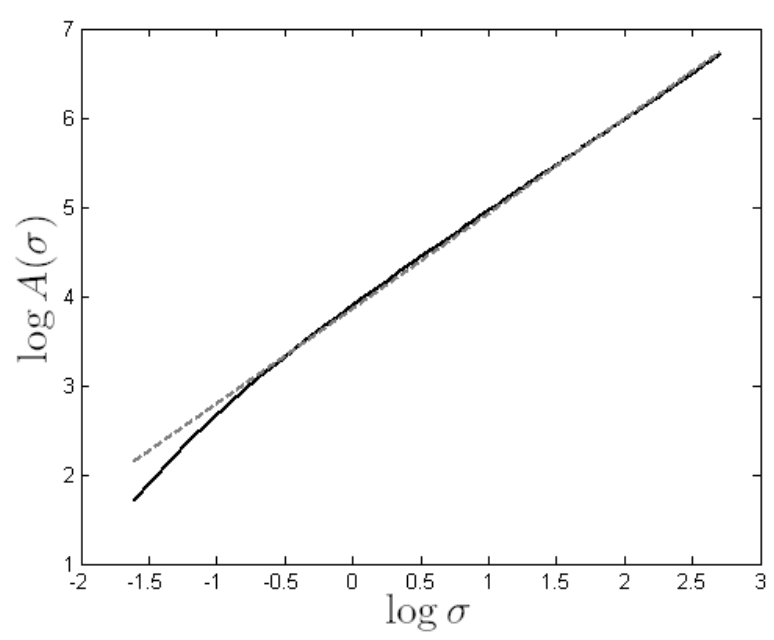

Fig. 2. Log-log curve and the computed line approximation

\section{Experiments}

Proposed descriptors were evaluated in a shape classification experiment which aimed to classify a set of shapes of plant's leaves. A total of 600 shapes of leaves, grouped into 30 classes with 20 samples each, were used. Figure 3 shows an example of each plant specie considered during the experiment. Leaves classification is a difficult task, due to the high inter-class similarity and the fact of the within-class similarity is unsuitable and overlaps can occur between adjacent parts of leaves.

Statistical analysis of the descriptors was performed by using the Linear Discriminant Analysis (LDA). This is a supervised classification method, which searches a linear sub-space where data projection presents variance larger intraclasses than inter-classes 12,13. The leave-one-out cross-validation strategy was also used over the LDA.

\section{Results}

As proposed in Section 3, leaves shapes are classified using a feature vector composed by a total of $n$ descriptors. Thus, it is necessary to define the value for $n$ that leads to a better discrimination of the samples. Figure 4 shows the success rate as a function of the number of descriptors used in the classification process. As expected, an increase in the success rate occurs as the number of 


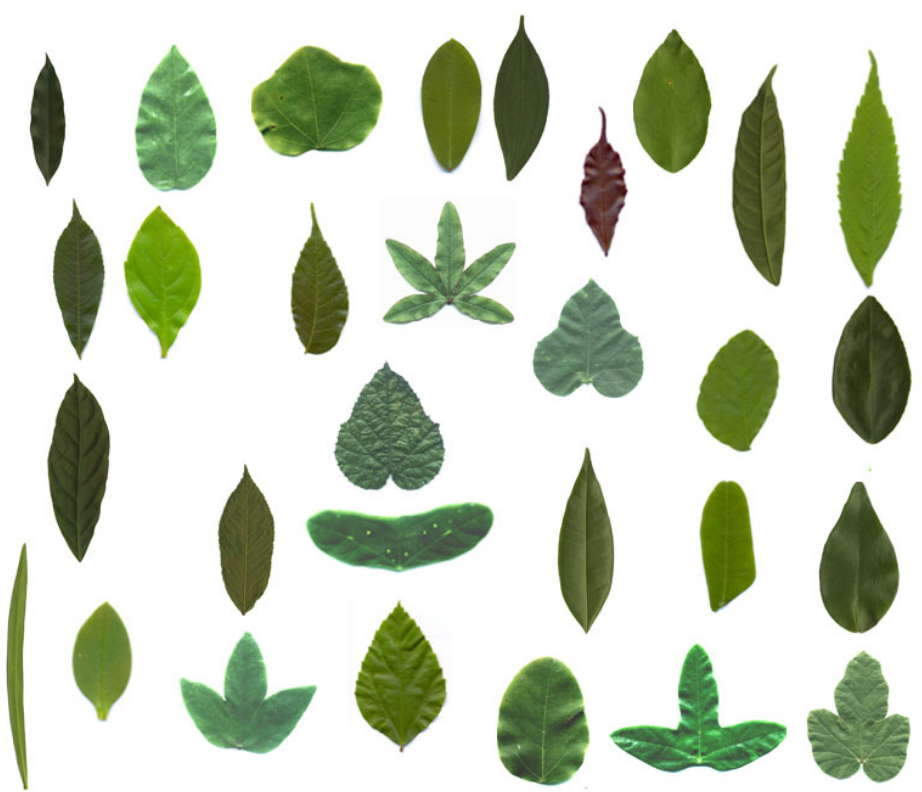

Fig. 3. Example of each leave class used in the experiments

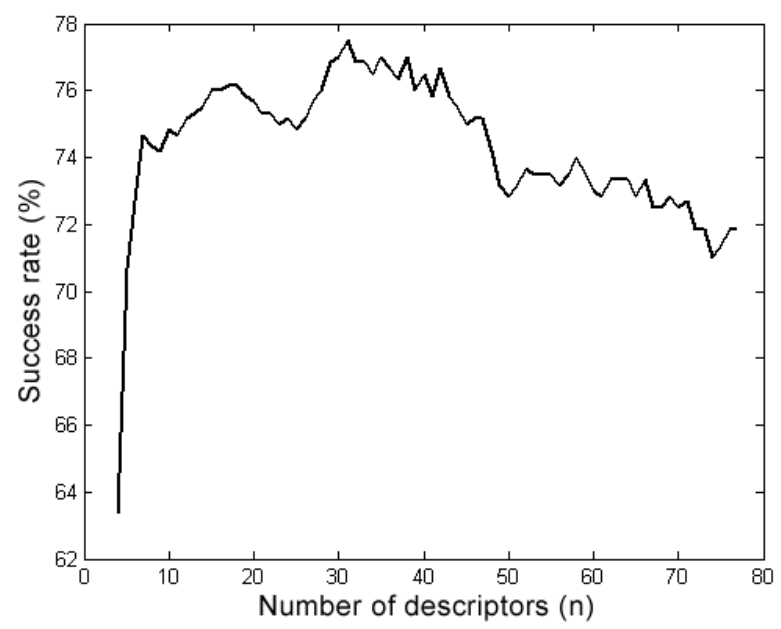

Fig. 4. Success rate according to the number of descriptors $(n)$ used

descriptors used is increased. This is a natural behavior as the increase of $n$ means the addition of more information about the shape complexity at different scales in the feature vector. However, relevant information for shape characterization is acquired until $n=31$. After this value, the information added to the feature vector is no longer relevant for shape discrimination and its use leads to misclassification of the samples and decrease of the success rate. 
In order to perform a better evaluation of the proposed approach, a comparison with traditional shape analysis methods was also performed. A brief description of each method is presented as follows:

Fourier descriptors: a feature vector is composed by selecting the 20 most significant coefficients computed from the application of the Fourier Transform over the shape contour 915.

Zernike moments: a feature vector containing 20 moments $(n=0,1, \ldots, 7)$ is used to represent each image. These moments are the most significant magnitudes of a set of orthogonal complex moments of the image [4.

Multi-scale Fractal Dimension: a curve which describes how the shape complexity changes as the scale changes is computed from the log-log curve estimated by using the Bouligand-Minkowski method. The 50 most meaningful points of the curve were considered for shape characterization [2]14].

Table 1. Classification performance of various shape descriptors

\begin{tabular}{|ccc|}
\hline Method & Samples correctly classified Success rate \\
\hline Proposed approach & 465 & 77.50 \\
Fourier descriptors & 450 & 75.00 \\
Zernike moments & 408 & 68.00 \\
M. S. Fractal Dimension & 438 & 73.00 \\
\hline
\end{tabular}

Table 1 presents the success rate achieved for each descriptor compared. A total of $n=31$ descriptors was used in our proposed method. Results indicate a higher performance of our approach when compared with Fourier descriptors, Zernike moments and Multi-scale Fractal Dimension. We must emphasize that leaves shapes classification is a difficult task. There is a high similarity interclasses of leaves while the similarity of intra-classes is low due to the presence of imperfections in the leaf which act as noise in the samples. This fact indicates that the proposed descriptors have a high capacity of discrimination between similar classes and also a high tolerance to within class variations, overcoming traditional shape analysis methods found in literature.

\section{Conclusion}

We presented a study of leaf shape classification based on curvature and complexity analysis. The classification of plant based exclusively on leaves is a very difficult task due to the high similarity inter-classes and low similarity of intraclasses. However, the leaf is an important characteristic of the plant which can be collected at any season or any stage of plant maturity, what justifies its choice in the experiments.

By using the Curvature Scale Space (CSS), we achieved a curve which enable us to describe the complexity of the shape. Descriptors extracted from this curve were used to classify a set of leaves shapes in an experiment using linear discriminant analysis. Results demonstrate the potential of the technique, which overcome traditional shape analysis methods found in literature. 


\section{Acknowledgments}

A.R.B. acknowledges support from FAPESP (2006/54367-9). J.B.F. acknowledges support from CNPq (870336/1997-5) and FAPESP (2006 / 53959-0). O.M.B. acknowledges support from CNPq (306628 / 2007-4).

\section{References}

1. Loncaric, S.: A survey of shape analysis techniques. Pattern Recognition 31(9), 983-1001 (1998)

2. da S. Torres, R., Falcão, A., da F. Costa, L.: A graph-based approach for multiscale shape analysis. Pattern Recognition 37(6), 1163-1174 (2003)

3. Wang, Z., Chi, Z., Feng, D.D.: Shape based leaf image retrieval. IEE ProceedingsVision Image and Signal Processing 150(1), 34-43 (2003)

4. Zhenjiang, M.: Zernike moment-based image shape analysis and its application. Pattern Recognition Letters 21(2), 169-177 (2000)

5. Osowski, S., Nghia, D.D.: Fourier and wavelet descriptors for shape recognition using neural networks - a comparative study. Pattern Recognition 35(9), 1949 1957 (2002)

6. Manoel, E.T.M., da F. Costa, L., Streicher, J., Muller, G.B.: Multiscale fractal characterization of three-dimensional gene expression data. In: SIBGRAPI, pp. 269-274. IEEE Computer Society, Los Alamitos (2002)

7. da F. Costa, L., Jr., R.M.C.: Shape Analysis and Classification: Theory and Practice. CRC Press, Boca Raton (2000)

8. Backes, A.R., Bruno, O.M.: A new approach to estimate fractal dimension of texture images. In: Elmoataz, A., Lezoray, O., Nouboud, F., Mammass, D. (eds.) ICISP 2008. LNCS, vol. 5099, pp. 136-143. Springer, Heidelberg (2008)

9. Gonzalez, R.C., Woods, R.E.: Digital Image Processing, 2nd edn. Prentic-Hall, New Jersey (2002)

10. Witkin, A.P.: Scale space filtering: a new approach to multi-scale descriptions. In: ICASSP - IEEE International Conference on Acoustics, Speech, and Signal Processing, GRETSI, pp. 79-95 (2003)

11. Mokhtarian, F., Abbasi, S.: Matching shapes with self-intersections: application to leaf classification. IEEE Transactions on Image Processing 13(5) (2004)

12. Everitt, B.S., Dunn, G.: Applied Multivariate Analysis, 2nd edn. Arnold (2001)

13. Fukunaga, K.: Introduction to Statistical Pattern Recognition, 2nd edn. Academic Press, London (1990)

14. de Plotze, R.O., Falvo, M., Pádua, J.G., Bernacci, L.C., Vieira, M.L.C., Oliveira, G.C.X., Bruno, O.M.: Leaf shape analysis using the multiscale minkowski fractal dimension, a new morphometric method: a study with passiflora (passifloraceae). Canadian Journal of Botany 83(3), 287-301 (2005) 